DOI: $10.3901 / J M E .2019 .10 .142$

\title{
窗型多壁结构的耐撞性研究*
}

\author{
何宁张勇 陈腾腾 林继铭 徐翔 \\ (华侨大学机电及自动化学院 厦门 361021)
}

\begin{abstract}
摘要: 具有高效吸能特性的薄壁结构已被广泛应用于各种运载工具中, 但传统单壁结构存在易陷入全局弯曲的不稳定吸能模 式。因此, 提出一种窗型多壁结构, 并采用试验测试、仿真分析与理论预测相结合的方法, 详细研究了窗型多壁结构、均匀 方形多胞结构与方形三壁结构在轴向载荷作用下的吸能特性。研究结果表明: 窗型多壁结构比同质量的另外两种结构具有更 高的吸能效率。同时, 窗型多壁结构的几何参数(壁厚 $T$ 、内层壁边长 $D$, 壁间距比 $N$ ) 对结构的耐撞性能有显著影响。并且, 基于超折叠单元理论开发了窗型多壁结构的理论模型，该模型能较好的预测窗型多壁结构的平均碰撞力与吸能特性，并采用 数值分析结果与理论预测结果的对比来验证了理论预测模型的可靠性。研究结果对开发新颖的轻量化薄壁吸能结构提供了良 好的指导与理论支撑。
\end{abstract}

关键词: 多壁结构; 能量吸能; 超折叠单元; 理论预测 中图分类号: U463

\section{Crashworthiness Study of Window Multi-wall Structure}

\section{HE Ning ZHANG Yong CHEN Tengteng LIN Jiming XU Xiang}

(College of Mechanical Engineering and Automation, Huaqiao University, Xiamen 361021)

\begin{abstract}
Thin-walled structures have been widely used in various delivery vehicles due to high energy absorption performance, but single-wall structures easily suffer global bending and cause unstable energy absorption mode. Therefore, a window multi-wall structure is proposed to improve the energy absorption of single wall structures. The energy absorption characteristics of the window multi-wall structure, uniform multi-cell square structure and square three-wall structure are detail investigated under axial loading by experimental testing, simulation analysis and theoretical prediction. The results show that the window multi-wall structure has higher energy absorption efficiency than other two structures of the same mass. At the same time, the geometric parameters, such as wall thickness $T$, inner wall side length $D$ and wall spacing ratio $N$, have a significant effect on crashworthiness of the window multi-wall structure. Furthermore, a theoretical model of the window multi-wall structure is developed to predict the mean crushing force and energy absorption of the window multi-wall structure based on super folding element theory. The reliability of the theoretical model is validated by comparing numerical results with theoretical solutions. The research findings offer a good guidance and theoretical support for development a novel thin-walled energy absorption structures.
\end{abstract}

Keywords: multi-wall structure; energy absorption; super folding element; theoretical prediction

\section{0 前言}

随着汽车保有量的逐年增长，交通事故的数量 也逐年攀升, 汽车的碰撞安全性能已经成为人们不 可忽视的问题。因此, 具有高的吸能性及吸能效率 的薄壁结构在车辆领域得到了广泛的应用 ${ }^{[1]}$, 甚至 在航天航空领域等轻量化设计中也发挥着重要的作 用。并且, 国内外学者已在薄壁结构的吸能性方面

\footnotetext{
* 国家自然科学基金(51675190)、福建省高等学校新世纪优秀人才计划和 华侨大学研究生科研创新基金资助项目。20180604 收到初稿, 20181212 收到修改稿
}

开展了许多研究工作。其中, 在单壁结构方面, WIERZBICKI 等 ${ }^{[2]}$ 研究了方管的轴向碰撞, 并提出 超折叠单元理论(Super folding element, SFE)来预测 其吸能。ABRAMOWICZ 等 ${ }^{[3]}$ 研究了圆柱管和方管 的轴向碰撞, 并推导出平均碰撞力的理论模型。但 是, 由于单壁结构仍存在轴向冲击载荷作用下的吸 能能力不足, 易出现欧拉屈曲现象等缺陷 ${ }^{[4]}$, 从而 使得薄壁管结构的吸能效率大大降低。

近年来，具有更好耐撞性能的多胞结构引起了 研究人员的高度关注。CHEN 等 ${ }^{[5]}$ 提出了简化超级 折叠单元理论 (Simplified super folding element, SSFE), 并推导出多胞管的折叠波长和平均载荷的 
理论表达式。ZHANG 等 ${ }^{[6]}$ 开展了相同质量的不同 方形多胞铝管的轴向碰撞吸能性分析, 发现具有更 多管壁的多壁结构能有效提高吸能效率。ANNISA 等 ${ }^{[7]}$ 通过数值分析和试验研究的手段对单壁, 双壁 及多壁管在动态轴向冲击力作用下的耐撞性进行了 研究, 结果表明在双壁管的壁间增加加强肋有利于 提高多胞管的耐撞性能。此外, WANG 等 ${ }^{[8]}$ 研究了 等质量不同胞体数的均匀方形多胞结构在轴向载荷 作用下的吸能性, 发现胞体数越多, 均匀方形多胞 结构的吸能性越强。并且, WU 等 ${ }^{[9]}$ 提出并研究了 一种 5 胞方形管结构耐撞性, 发现 5 胞结构的吸能 性明显优于 4 胞方形结构和单壁方管, 进一步表明 增加胞元数能有效提高吸能效率。

上述研究发现, 薄壁结构的多壁特性能较好的 改善其吸能性, 是薄壁结构推进和发展的一个重要 方向, 对发掘薄壁结构的潜在吸能特性具有很高的 研究价值。综合上述研究, 薄壁结构的塑性应变及 能量耗散主要集中于结构的拐角区域 ${ }^{[2]}$, 且拐角区 域形成 $90^{\circ}$ 夹角能有效提高结构的平均碰撞力和 能量吸收 ${ }^{[10-11]}$ 。因此, 基于这些发现的启发, 提出 一种窗型多壁结构, 并采用试验、数值分析与理论 推导方法详细的讨论了该结构的吸能性和耐撞性。 研究结果对开发新颖的轻量化薄壁吸能结构提供了 良好的指导与理论支撑, 也对我国汽车被动安全性 及轻量化研究的发展具有重要的现实意义。

\section{1 窗型多壁结构的数值模型}

\section{1 几何模型}

窗型多壁结构(Window multi-walled column tube, $\mathrm{WMCT}$ )的几何截面及其几何参数如图 1a 所示, 它们 分别是壁厚 $T$, 内层壁边长 $D$, 壁间距比 $N\left(N=d_{1} / d_{2}\right)$ 。 $d_{1}, d_{2}$ 分别为外壁到中壁的距离和中壁到内壁的距 离。并且, 为了开展对比研究, 建立均匀方形多胞 $(3 \times 3)$ 结构(Uniform multi-cell square tube, UMST)与方形三 壁结构(Square three-walled tube, STT)的几何截面及 其几何参数如图 $1 \mathrm{~b}$ 和图 $1 \mathrm{c}$ 所示, 各个方形截面的

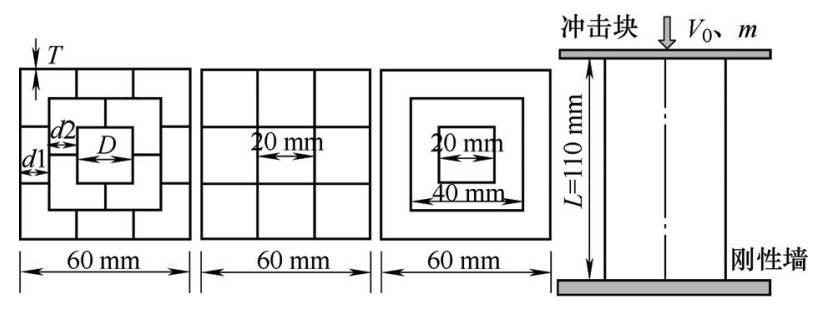
(a) WMCT
(b) UMST
(c) STT
(d) 示意图

图 1 窗型多壁结构的几何截面及碰撞示意图
边长皆为 $60 \mathrm{~mm}$ 。为了研究各个结构的耐撞性, 图 $1 \mathrm{~d}$ 建立了三种多壁结构的冲击示意图。其长度为 $L=110 \mathrm{~mm}$, 底部固定在刚性墙上。质量 $m=300 \mathrm{~kg}$ 的冲击块以 $V_{0}=20 \mathrm{~m} / \mathrm{s}$ 的初始速度冲击多壁结构。

\section{2 多壁结构的有限元模型}

基于图 1 的几何模型, 在非线性有限元软件 LS-DYNA 建立了三种多壁结构的有限元碰撞模型 如图 2, 多壁管采用 $2 \mathrm{~mm} \times 2 \mathrm{~mm}$ 的 BT 四节点壳单 元进行离散, 冲击块与刚性墙定义为刚体。为了模 拟多壁结构与刚性支撑之间的接触力, 多壁管和刚 性支撑之间采用自动点面接触, 为避免多壁管在压 缩过程中自身的接触穿透, 多壁管采用自动单面接 触, 且接触的静、动摩擦因数分别取为 0.3 和 0.2 。

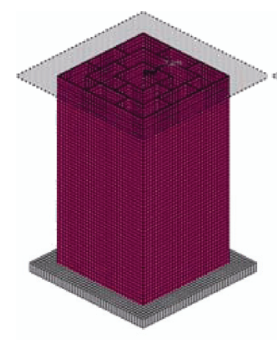

(a) WMCT

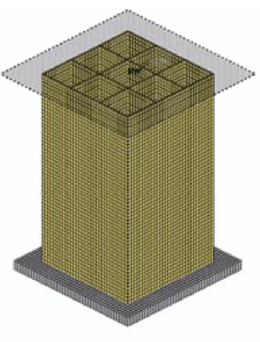

(b) UMST

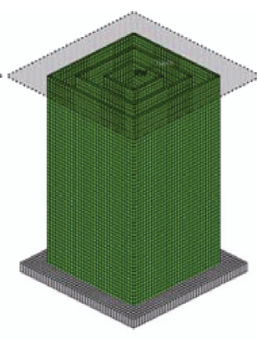

(c) STT
图 2 有限元模型

多壁管采用 LS-DYNA 中的 MAT-24 号材料模 型, 其材料为铝合金 A16061-O, 其基本材料参数如 下: 密度 $\rho=2.7 \times 10^{3} \mathrm{~kg} / \mathrm{m}^{3}$, 弹性模量 $E=68.2 \mathrm{GPa}$, 初始屈服应力 $\sigma_{\mathrm{y}}=96.8 \mathrm{MPa}$, 极限应力 $\sigma_{u}=195 \mathrm{MPa}$, 泊松比 $\mu=0.3$, 图 3 为 A16061-O 建立的拉伸试件及得 到的有效应力应变曲线。刚性支撑采用 LS-DYNA 中 的 MAT-20 号刚性材料模型。此外, 由于铝合金应变 率不敏感, 在数值模型中忽略了应变率效应 ${ }^{[12]}$ 。

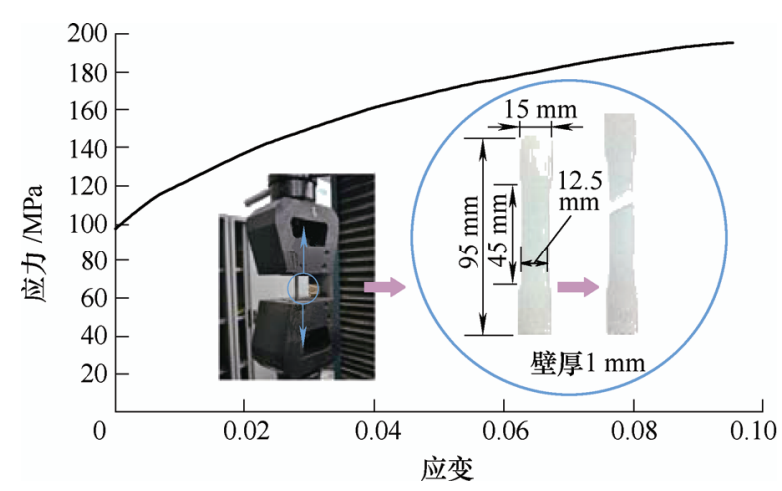

图 3 A16061-O 有效应力应变曲线

\section{3 试验分析与数值模型验证}

为了获取多壁结构的吸能特性和验证有限元模 型的可行性。首先基于线切割方法加工了如图 4a 所 示为壁厚为 $0.6 \mathrm{~mm}$ 的 UMST 试验试件, UMST 试件 在 MTS 万能材料试验机开展了速度为 $5 \mathrm{~mm} / \mathrm{min}$ 的 
准静态试验。其在 $70 \mathrm{~mm}$ 的压缩位移的变形如图 $4 \mathrm{~b}$ 所示, UMST 有较好的逐级折叠的变形模式。

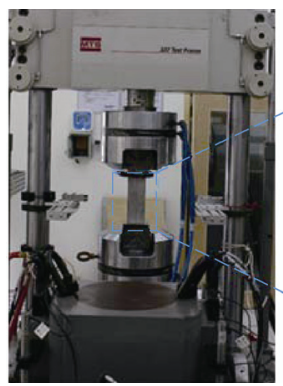

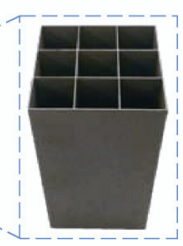

(a)

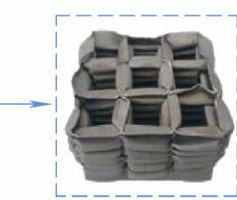

(b)
图 4 在 MTS 万能试验机上进行准静态试验

此外, 为了验证文章数值模型的可行性, 基于 试验的加载边界条件, 对 UMST 结构开展了仿真分 析, 图 5a 分别给出了不同压缩位移下试验与仿真的 变形模式对比, 由图可知, 试验与仿真的变形模式 具有较好的一致性, 表明了数值分析结果的准确性。 图 5b 进一步给出了 UMST 结构试验与仿真的压缩 力-位移曲线, 其中试验与仿真曲线在波峰与波谷的 波动性上具有较好的一致性, 从而进一步表明建立 的有限元数值模型的准确性与可靠性。
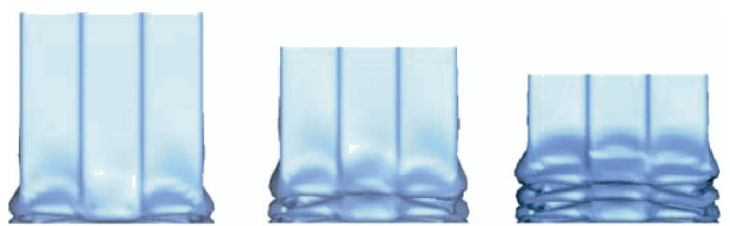

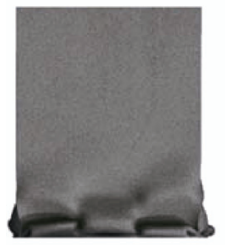

$10 \mathrm{~mm}$

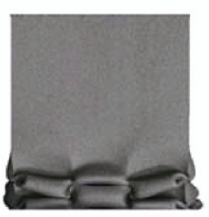

$30 \mathrm{~mm}$

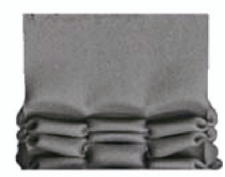

$50 \mathrm{~mm}$ (a) 不同压缩位移下试验与仿真的变形模式对比

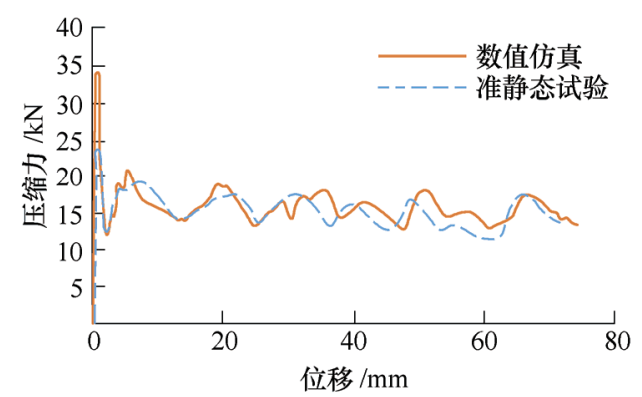

(b) 试验与仿真的压缩力 - 位移曲线

图 5 试验测试与有限元模型的比较

\section{2 数值结果与讨论}

\section{1 耐撞性指标}

多壁结构的主要耐撞性指标包括总吸能量
(Energy absorption, EA), 比吸能(Specific energy absorption, SEA), 峰值碰撞力 (Peak crushing force, PCF), 碰撞力效率(Crushing force efficiency, CFE)等。

SEA 是最关键的能量吸能效率指标, 其表示了 多壁结构单位质量的吸能率 ${ }^{[13]}$ ，其可表示为

$$
\mathrm{SEA}=\frac{\mathrm{EA}}{m}
$$

式中, $m$ 表示结构的质量, EA 表示结构在塑性变形 过程中的总吸能量, 其方程为

$$
\mathrm{EA}=\int_{0}^{\delta} F(x) \mathrm{d} x
$$

式中, $\delta$ 表示有效压缩位移, 所提出的有效压缩位 移为 $70 \mathrm{~mm} 。 F(x)$ 是碰撞过程中的瞬时碰撞力, $F(x)$ 的最大值(Peak crushing force, PCF)和平均值(Mean crushing force, MCF)与乘员的安全密切相关, 因为 当碰撞发生时高的 PCF 会导致高的冲击加速度以及 高的伤亡率, MCF 可表示为

$$
\mathrm{MCF}=\frac{\mathrm{EA}}{\delta}=\frac{\int_{0}^{\delta} F(x) \mathrm{d} x}{\delta}
$$

另外, 碰撞力效率 CFE 也是一个很重要的指标, 它表示冲击过程中的载荷的稳定性, CFE 可表示为

$$
\mathrm{CFE}=\frac{\mathrm{MCF}}{\mathrm{PCF}} \times 100 \%
$$

CFE 值越高表明载荷稳定性就越高 ${ }^{[14]}$ 。

\section{2 多壁与多胞结构的耐撞性对比}

为了研究三种多壁结构的耐撞性能, 首先分析 三种多壁结构在 5 种不同质量下的耐撞性，表 1 给 出了各质量参数及其对应的耐撞性指标值。其中,

表 1 不同质量下三种多壁结构的数值结果

\begin{tabular}{ccccccc}
\hline 多壁结构 & 质量 $/ \mathrm{kg}$ & 壁厚 $/ \mathrm{mm}$ & EA $/ \mathrm{kJ}$ & $\mathrm{SEA} /(\mathrm{kJ} / \mathrm{kg})$ & $\mathrm{PCF} / \mathrm{kN}$ & $\mathrm{CFE}$ \\
\hline $\mathrm{WMCT}_{1}$ & 0.107 & 0.60 & 2.300 & 21.497 & 51.635 & 0.636 \\
$\mathrm{UMST}_{1}$ & 0.107 & 0.75 & 1.986 & 18.580 & 51.714 & 0.549 \\
$\mathrm{STT}_{1}$ & 0.107 & 0.75 & 1.287 & 12.042 & 51.485 & 0.357 \\
$\mathrm{WMCT}_{2}$ & 0.160 & 0.90 & 4.430 & 27.616 & 83.443 & 0.758 \\
$\mathrm{UMST}_{2}$ & 0.160 & 1.13 & 3.740 & 23.376 & 84.182 & 0.635 \\
$\mathrm{STT}_{2}$ & 0.160 & 1.13 & 2.478 & 15.487 & 82.203 & 0.431 \\
$\mathrm{WMCT}_{3}$ & 0.214 & 1.20 & 6.874 & 32.149 & 116.267 & 0.845 \\
$\mathrm{UMST}_{3}$ & 0.214 & 1.50 & 5.811 & 27.181 & 117.964 & 0.704 \\
$\mathrm{STT}_{3}$ & 0.214 & 1.50 & 3.877 & 18.133 & 113.727 & 0.487 \\
$\mathrm{WMCT}_{4}$ & 0.267 & 1.50 & 9.555 & 35.745 & 167.681 & 0.814 \\
$\mathrm{UMST}_{4}$ & 0.267 & 1.88 & 8.280 & 30.975 & 154.738 & 0.764 \\
$\mathrm{STT}_{4}$ & 0.267 & 1.88 & 5.943 & 22.232 & 145.978 & 0.582 \\
$\mathrm{WMCT}_{5}$ & 0.321 & 1.80 & 12.334 & 38.447 & 219.123 & 0.804 \\
$\mathrm{UMST}_{5}$ & 0.321 & 2.26 & 11.021 & 34.355 & 209.119 & 0.753 \\
$\mathrm{STT}_{5}$ & 0.321 & 2.26 & 7.680 & 23.940 & 178.656 & 0.614 \\
\hline
\end{tabular}


窗型多壁结构的内层壁边长 $D$ 与壁间距比 $N$ 分别为 $20 \mathrm{~mm}$ 与 1 。

图 6 表示 3 种多壁结构在 5 组不同质量下的 SEA 柱状图。首先, 三种结构的 SEA 都随着质量的 增加而增加, 其中, WMCT 具有明显吸能性优势, $\operatorname{WMCT}(m=0.214 \mathrm{~kg})$ 的 SEA 和 CFE 分别比同质量的 UMST 提升了 $18 \%$ 和 $20 \%$, 也比同质量的 STT 的 SEA 和 CFE 分别提升了 $77 \%$ 和 $73 \%$, 且 STT 结构 在各种质量参数下都具有最低的 SEA。图 7 进一步 给出了三种结构 $(m=0.214 \mathrm{~kg})$ 在压缩位移 $70 \mathrm{~mm}$ 处 的压缩力-位移曲线及对应的变形模式, 由图可知, WMCT 与 UMST 都发生了较好的逐级折叠的变形 模式, 而 STT 却发生了不规则的变形模式。并且, WMCT 的压缩力-位移曲线明显高于 UMST。

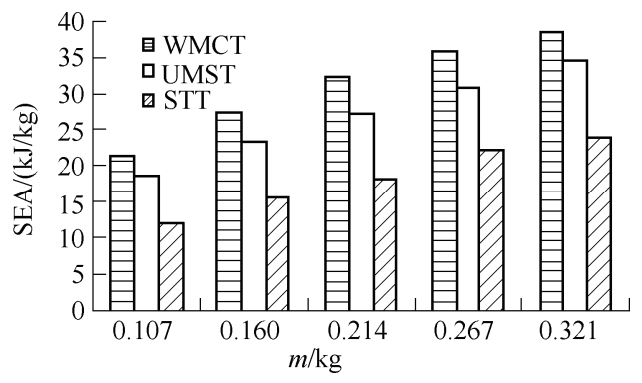

图 6 三种多壁结构的 SEA

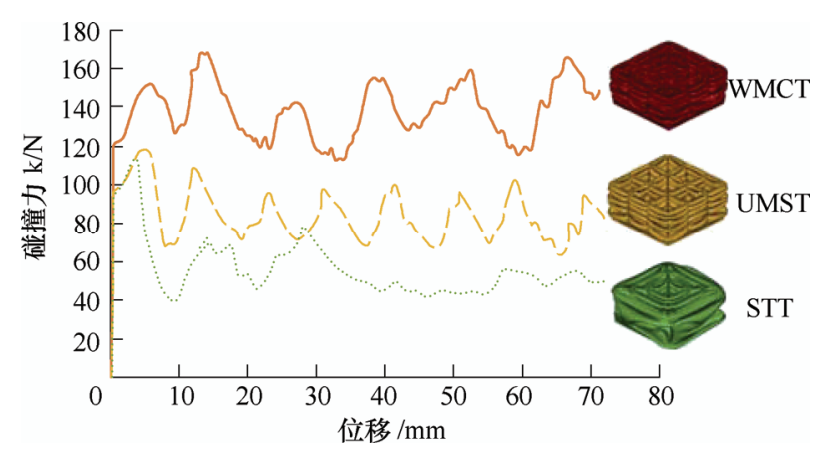

图 7 三种多壁结构的压缩力与位移曲线

图 8 给出了 3 种多壁管在 5 组质量下的 $\mathrm{CFE}$ 。 由图可知, $\mathrm{WMCT}$ 结构在不同的质量下都具有最高 的 CFE，表明其具有最好的载荷稳定性。此外，三 种结构的 CFE 随着质量的增加都呈现出先增后降 的趋势, 从而表明增加结构的质量并不总能提高结 构的载荷一致性。

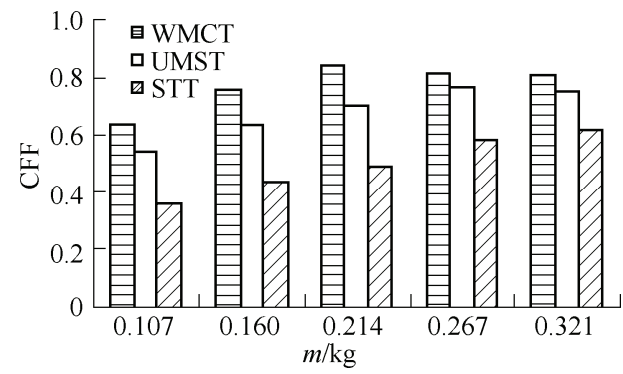

图 8 三种多壁结构的 CFE 对比
综上可知, WMCT 结构在能量吸收能力与载荷 稳定性方面都优于 UMST 和 STT。因此, 所提出的 窗型多壁结构具有优异的耐撞性。

\section{3 窗型多壁结构的耐撞性参数研究}

虽然 WMCT 具有较好的能量吸收性能, 但其 耐撞性也与其几何拓扑参数息息相关。因此，进一 步开展了 $\mathrm{WMCT}$ 在不同几何参数的耐撞性研究。

\subsection{1 内层壁边长 $D$ 和壁厚 $T$ 的影响}

在保持壁间距比 $N=1$ 的情况下, 表 2 给出了 $D$ 与 $T$ 的 2 因素 5 水平全因子设计的耐撞性参数 及其指标。

表 2 不同 $\boldsymbol{D}$ 与 $\boldsymbol{T}$ 下的 WMCT 的耐撞性指标

\begin{tabular}{|c|c|c|c|c|c|}
\hline 壁间距/mm & 壁厚/mm & $\mathrm{EA} / \mathrm{kJ}$ & $\mathrm{SEA} /(\mathrm{kJ} / \mathrm{kg})$ & $\mathrm{PCF} / \mathrm{kN}$ & CFE \\
\hline 10 & 0.6 & 2.291 & 22.548 & 49.022 & 0.668 \\
\hline 10 & 0.9 & 4.396 & 28.848 & 78.025 & 0.805 \\
\hline 10 & 1.2 & 6.906 & 33.904 & 114.714 & 0.860 \\
\hline 10 & 1.5 & 9.829 & 38.714 & 161.203 & 0.871 \\
\hline 10 & 1.8 & 13.217 & 43.377 & 221.392 & 0.853 \\
\hline 15 & 0.6 & 2.337 & 22.431 & 50.302 & 0.664 \\
\hline 15 & 0.9 & 4.444 & 28.414 & 80.819 & 0.786 \\
\hline 15 & 1.2 & 7.014 & 33.642 & 115.243 & 0.870 \\
\hline 15 & 1.5 & 9.927 & 38.094 & 167.849 & 0.845 \\
\hline 15 & 1.8 & 12.936 & 41.370 & 216.565 & 0.853 \\
\hline 20 & 0.6 & 2.300 & 21.497 & 51.635 & 0.636 \\
\hline 20 & 0.9 & 4.430 & 27.616 & 83.443 & 0.758 \\
\hline 20 & 1.2 & 6.874 & 32.149 & 116.267 & 0.845 \\
\hline 20 & 1.5 & 9.555 & 35.745 & 167.681 & 0.814 \\
\hline 20 & 1.8 & 12.334 & 38.447 & 219.123 & 0.804 \\
\hline 25 & 0.6 & 2.378 & 21.696 & 53.144 & 0.639 \\
\hline 25 & 0.9 & 4.529 & 27.547 & 85.924 & 0.753 \\
\hline 25 & 1.2 & 6.772 & 30.892 & 119.911 & 0.807 \\
\hline 25 & 1.5 & 8.982 & 32.780 & 164.822 & 0.778 \\
\hline 25 & 1.8 & 11.303 & 34.378 & 212.855 & 0.759 \\
\hline 30 & 0.6 & 2.367 & 21.080 & 54.673 & 0.619 \\
\hline 30 & 0.9 & 4.396 & 26.103 & 88.268 & 0.711 \\
\hline 30 & 1.2 & 6.193 & 27.584 & 123.437 & 0.717 \\
\hline 30 & 1.5 & 8.429 & 30.030 & 161.626 & 0.745 \\
\hline 30 & 1.8 & 10.651 & 31.624 & 200.516 & 0.759 \\
\hline
\end{tabular}

图 9 表示 $\mathrm{WMCT}$ 在不同 $D$ 与 $T$ 值下 $\mathrm{SEA}$ 曲线,

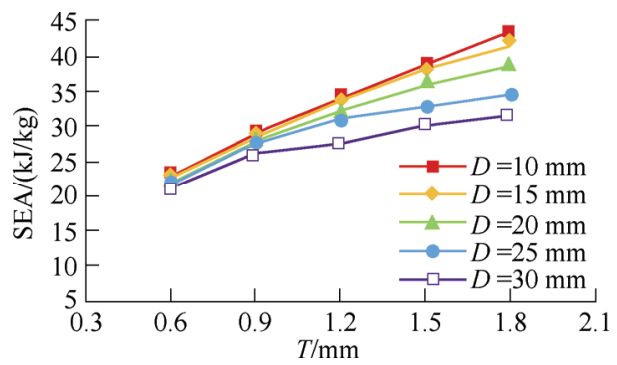

图 9 SEA 随 $D$ 与 $T$ 的变化曲线 
逐渐降低的趋势。图 10 进一步给出了 $\mathrm{WMCT}(T=1.2$ $\mathrm{mm}$ )在不同 $D$ 值下的变形模式, 由图 10 可知, SEA 随着 $T$ 的增加而增加, 但曲线的增长趋势逐渐减小。 并且, 随着 $D$ 值增大, SEA 呈现随着 $D$ 值的增加, $\mathrm{WMCT}$ 的折叠波长逐渐增大, 折叠数逐渐减少, 从 而导致 SEA 的下降。
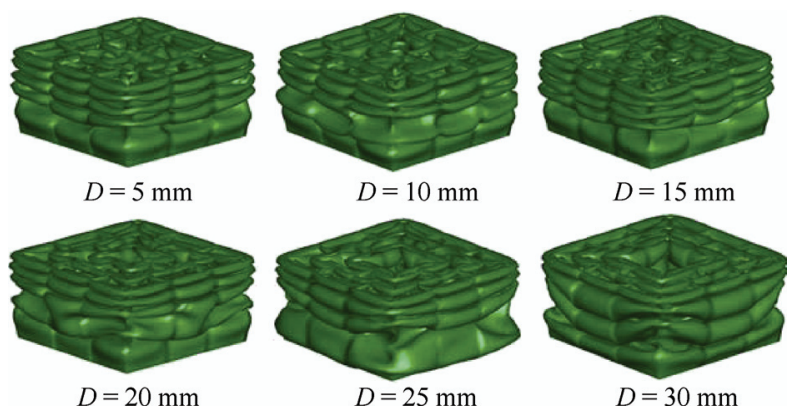

图 $10 \operatorname{WMCT}(T=1.2 \mathrm{~mm}, N=1)$ 的变形模式

另外, 图 11 表明 $\mathrm{PCF}$ 随 $T$ 增长呈现明显的增 大趋势, 表明 PCF 对结构厚度的变化具有非常强的 敏感度。且不同 $D$ 值对应的 PCF 曲线基本重合, 表 明 $\mathrm{PCF}$ 对内层壁边长 $D$ 不敏感。CFE 随 $D$ 与 $T$ 变 化的曲线如图 12 所示, CFE 呈现了明显的先增后 降的趋势, 且在 $T=1.2 \mathrm{~mm}$ 附近出现最大值。且随 着 $D$ 的增大, CFE 也呈现下降的趋势。由此可知, $T$ 与 $D$ 皆对 WMCT 的耐撞性能有重要影响, 且增 大 $D$ 不利于改善 $\mathrm{WMCT}$ 的吸能性。

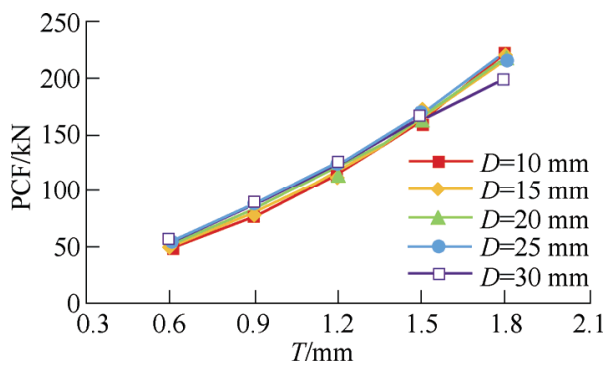

图 $11 \mathrm{PCF}$ 随 $D$ 与 $T$ 的变化曲线

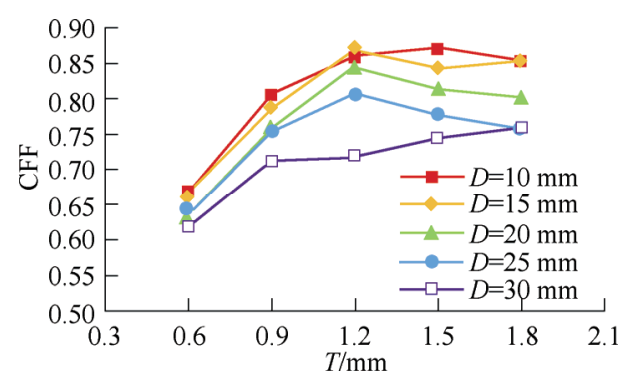

图 $12 \mathrm{CFE}$ 随 $D$ 与 $T$ 的变化曲线

\subsection{2 壁间距比 $N$ 的影响}

本节在保持内层壁边长 $D=20 \mathrm{~mm}$ 的情况下, 研究了壁间距比 $N$ 与对 WMCT 结构的耐撞性影响。 表 3 给出了在 3 组不同厚度的 WMCT 在不同 $N$ 下 的各耐撞性指标。
表 3 不同 $N$ 下的 WMCT 的耐撞性指标

\begin{tabular}{ccccccc}
\hline 壁厚 $/ \mathrm{mm}$ & 壁间距比 & 质量 $\mathrm{kg}$ & $\mathrm{EA} / \mathrm{kJ}$ & $\mathrm{SEA} / \mathrm{kJ} / \mathrm{kg})$ & PCF $/ \mathrm{kN}$ & $\mathrm{CFE}$ \\
\hline 0.9 & 0.5 & 0.164 & 4.303 & 26.253 & 85.888 & 0.716 \\
0.9 & 0.75 & 0.162 & 4.509 & 27.853 & 84.527 & 0.762 \\
0.9 & 1 & 0.160 & 4.430 & 27.616 & 83.443 & 0.758 \\
0.9 & 1.50 & 0.158 & 4.315 & 27.273 & 81.744 & 0.754 \\
0.9 & 2 & 0.157 & 4.160 & 26.533 & 80.433 & 0.739 \\
0.9 & 3 & 0.155 & 3.931 & 25.358 & 79.056 & 0.710 \\
1.2 & 0.50 & 0.219 & 6.347 & 29.033 & 120.007 & 0.756 \\
1.2 & 0.75 & 0.216 & 6.822 & 31.596 & 117.911 & 0.826 \\
1.2 & 1 & 0.214 & 6.874 & 32.149 & 116.267 & 0.845 \\
1.2 & 1.50 & 0.211 & 6.783 & 32.145 & 114.029 & 0.850 \\
1.2 & 2 & 0.209 & 6.601 & 31.568 & 113.350 & 0.832 \\
1.2 & 3 & 0.207 & 5.968 & 28.873 & 110.953 & 0.768 \\
1.5 & 0.50 & 0.273 & 8.895 & 32.559 & 156.974 & 0.810 \\
1.5 & 0.75 & 0.270 & 9.202 & 34.106 & 154.448 & 0.851 \\
1.5 & 1 & 0.267 & 9.555 & 35.745 & 155.025 & 0.880 \\
1.5 & 1.50 & 0.264 & 9.671 & 36.674 & 160.471 & 0.861 \\
1.5 & 2 & 0.261 & 9.360 & 35.806 & 160.693 & 0.832 \\
1.5 & 3 & 0.258 & 8.523 & 32.982 & 151.041 & 0.806 \\
\hline & & & & & &
\end{tabular}

其中, 图 13 与图 14 分别表示了 SEA 与 CFE 随 $N$ 变化的曲线, SEA 与 CFE 随 $N$ 都呈现先增大 后减小的趋势, 且约在 $N=1.5$ 时出现峰值。此外, 随着 $N$ 值的增加 PCF 有轻微的降低(图 15), 这是由

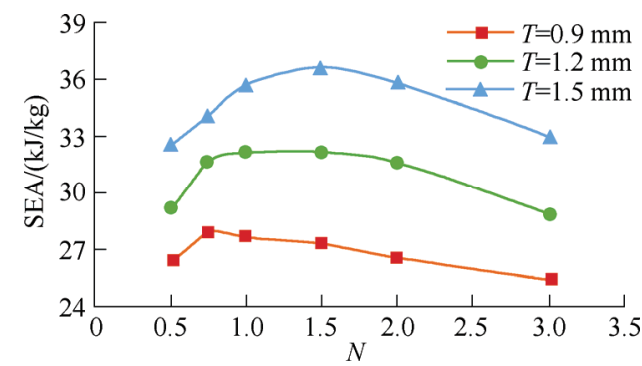

图 13 SEA 随 $N$ 的变化曲线

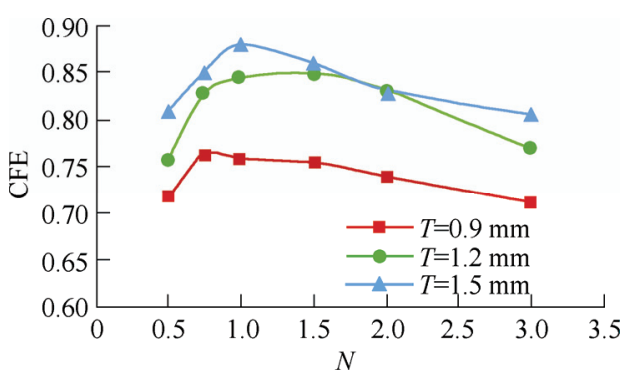

图 $14 \mathrm{CFE}$ 随 $N$ 的变化曲线

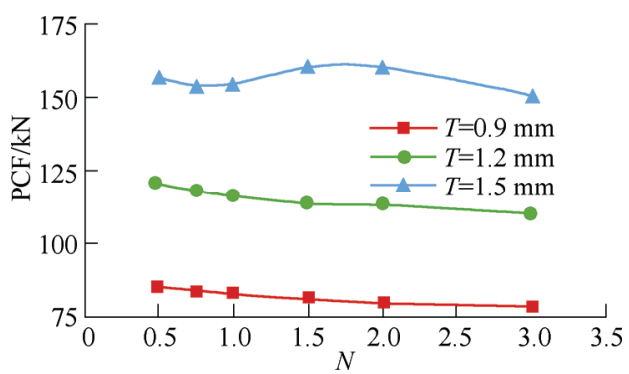

图 $15 \mathrm{PCF}$ 随 $N$ 的变化曲线 
于 $N$ 值的增加会使得 WMCT 的横截面积和刚度有 一定的减小，导致 PCF 降低。表明 $N$ 对 WMCT 的 耐撞性也有重要的影响。

\section{3 窗形多壁结构的理论预测}

理论预测能较好的获得结构的材料与几何参数 与结构变形和吸能性的数学关系, 是吸能结构设计 主要手段之一。因此, 进一步对 WMCT 开展理论 分析和预测的研究, 并获得 $N 、 D 、 T$ 与结构的吸能 性的理论预测模型。

在冲击载荷的作用下，薄壁结构被压溃产生折 叠的变形能等于弯曲变形能与薄膜变形能之和。因 此, 薄壁结构的能量吸收可表示为 ${ }^{[15]}$

$$
\mathrm{MCF} \cdot 2 H=\frac{1}{\eta}\left(E_{b}+E_{m}\right)
$$

式中, $\mathrm{MCF}$ 为平均碰撞力、 $2 H$ 为折叠波长、 $E_{\mathrm{b}}$ 为 弯曲变形能、 $E_{\mathrm{m}}$ 为薄膜变形能, $\eta$ 为有效压缩距离 系数。

\section{1 弯曲变形能}

基于简化超级折叠单元理论(Simplified super folding element, SSFE) ${ }^{[6]}$, 每个面的弯曲变形耗散 能可通过叠加弯曲铰链线得到, 每个基本折叠单元 有 3 个固定的弯曲铰链, 如图 16 给出了基本折叠单 元弯曲变形的折叠过程。

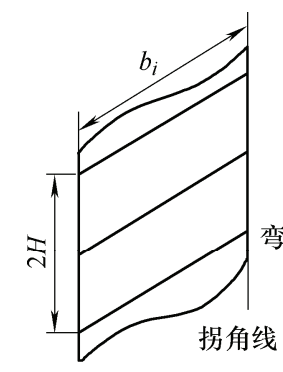

(a) 变形前

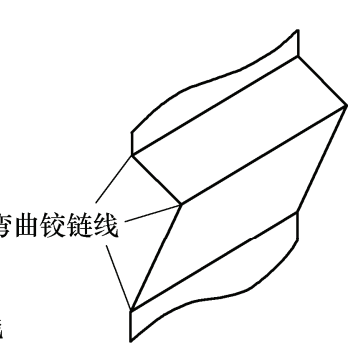

(b) 变形后

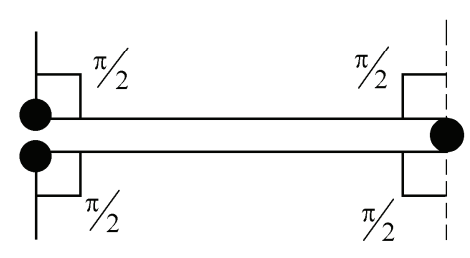

(c) 完全变形后

图 16 基本折叠单元弯曲变形的折叠过程 ${ }^{[16]}$

弯曲变形能 $E_{\mathrm{b}}$ 可表示为

$$
E_{b}=\sum_{i=1}^{m} \mu_{0} M_{0} b_{i}
$$

式中, $\mu_{0}$ 表示弯曲过程中铰链线的旋转角度。为了 简便, 假设在轴向压缩过程中, 基本折叠单元完全
折叠, 此时, 如图 $16 \mathrm{c}$ 弯曲铰链处的 4 个旋转角 $\mu$ 均为 $\pi / 2^{[6]}, b_{i}$ 表示单元的边长, $M_{0}=\sigma_{0} T^{2} / 4$ 是 薄壁发生塑性弯曲的弯矩, $\sigma_{0}$ 表示材料的流动 应力 ${ }^{[16-17]}$

$$
\sigma_{0}=\frac{\sigma_{u+} \sigma_{\mathrm{y}}}{2}
$$

假设每个面的作用相似, 则总的弯曲变形能可 用式(8)表示

$$
E_{b}=2 \pi M_{0} B
$$

式中, $B$ 为组成各个单元的边长之和, 即多胞结构 横截面周长。

\section{2 薄膜变形能}

根据 SSFE 理论, 薄膜变形能 $E_{\mathrm{m}}$ 是通过计算各 个拐角单元所耗散的能量和求得。图 17 给出了组成 WMCT 的两种基本拐角单元: L 形单元(圆圈)和 $\mathrm{T}$ 形单元(方形框)。

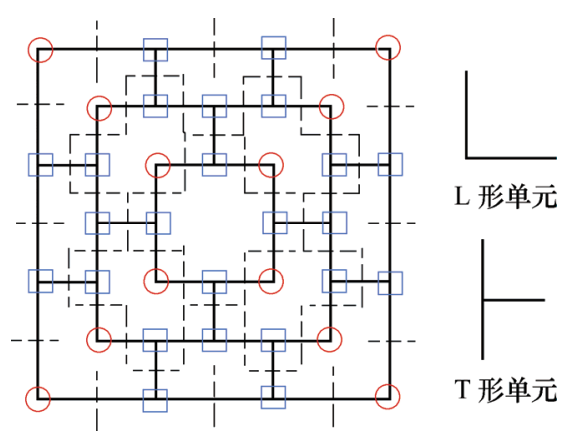

图 17 WMCT 的角单元示意图

\subsubsection{L 形单元的薄膜变形能}

图 18 表示 WMCT 中的 L 形单元(图 18a)和 T 形单元(图 18b)的折叠模式, 因此, L 形单元的变形 模式可表示为图 $19 \mathrm{a}^{[5]}$ 的对称模式, 两个面同时发生 折叠形成对称的三角形单元, 其三角形阴影区为能 耗区域。

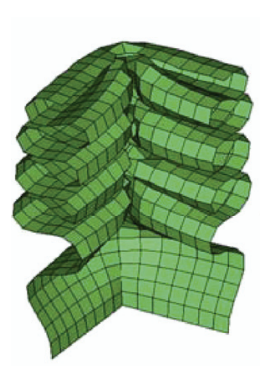

(a) L 形单元

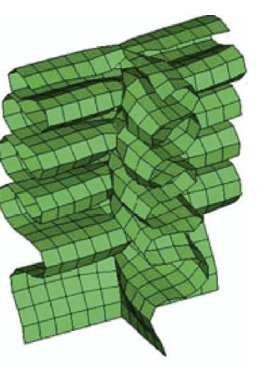

(b) $\mathrm{T}$ 形单元
图 $18 \mathrm{~L}$ 形单元和 $\mathrm{T}$ 形单元的变形模式

计算三角形阴影区域面积即得对称模式折叠的 膜变形能 $E_{\mathrm{m}}^{\mathrm{sym}[11]}$, 可以表示为

$$
E_{\mathrm{m}}^{\mathrm{sym}}=\int \sigma_{0} T \mathrm{~d} s=4 M_{0} \frac{H^{2}}{T}
$$


式中, $T$ 表示多壁管的厚度。

由于折叠过程中每个面的作用相似, 因此, 对 称模式下的 $\mathrm{L}$ 形单元的膜变形能 $E_{m}^{L}$ 相当于 2 倍 $E_{m}^{\text {sym }}$ ，表示为

$$
E_{\mathrm{m}}^{\mathrm{L}}=2 E_{\mathrm{m}}^{\mathrm{sym}}=8 M_{0} \frac{H^{2}}{T}
$$

\subsection{2 $\mathrm{T}$ 形单元的膜变形能}

如图 $18 \mathrm{bT}$ 形单元相当于在一个非对称的 L 形 单元的基础上添加一个面组合而成的。非对称 L 形 单元如图 $19 \mathrm{~b}$, 两个面发生折叠变形挤压后形成 3 个非对称的三角形单元。图 20 表示了三面折叠单元 的折叠过程和能量耗散区域 ${ }^{[18]}$, 图 $20 \mathrm{a}$ 为折叠过程, 图中 $\varphi$ 为面与面之间的夹角, $\mathrm{AME}$ 区域沿着路径 $O N$ 变形为 $A M N(E$ 点的初始位置位于 $O)$ 。图 20b 中的三角形区域即为能量耗散区域。因此, $\mathrm{T}$ 形单 元添加面的薄膜变形能 $E_{\mathrm{m}}^{a}$ 可表示为 ${ }^{[18]}$

$$
\begin{gathered}
E_{\mathrm{m}}^{a}=\int \sigma_{0} T \mathrm{~d} s=2 \sigma_{0} T H^{2} \tan (\varphi / 2)= \\
8 M_{0} \tan (\varphi / 2) \frac{H^{2}}{T}
\end{gathered}
$$

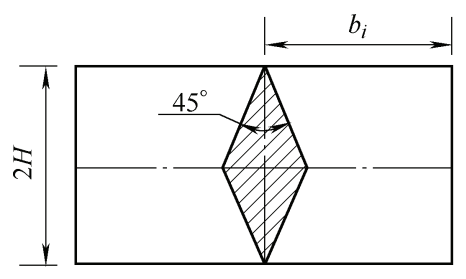

(a) 对称模式

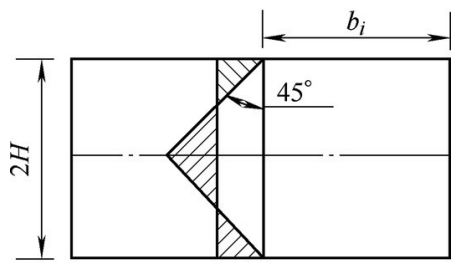

(b) 非对称模式

图 19 基础折叠单元模式

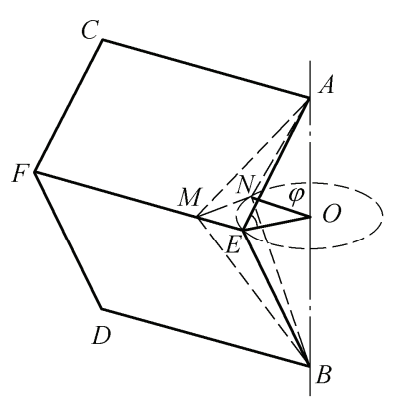

(a) 折叠过程

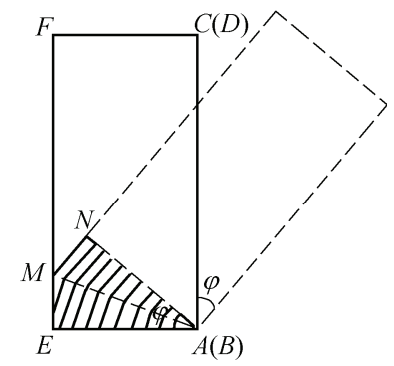

(b) 能量耗散区域
图 20 三面折叠单元的折叠过程和能量耗散区域

非对称模式折叠的薄膜变形能 $E_{m}^{a s y m}$ 可以表 示为

$$
E_{m}^{a s y m}=\int \sigma_{0} T \mathrm{~d} s=2 M_{0} \frac{H^{2}}{T}
$$

因此, $\mathrm{T}$ 形单元 $(\varphi=\pi / 4)$ 的薄膜变形能 $E_{\mathrm{m}}^{\mathrm{T}}$ 可以表 示为

$$
E_{\mathrm{m}}^{\mathrm{T}}=4 M_{0} \frac{H^{2}}{T}+8 M_{0} \frac{H^{2}}{T}=12 M_{0} \frac{H^{2}}{T}
$$

\subsection{WMCT 的 MCF 和 SEA 的理论模型}

WMCT 结构是由图 17 的 12 个 L 形单元和 24 个 $\mathrm{T}$ 形单元组成。因此, 将式(8)、(10)和(13)代入 式(5), 得到 WMCT 的平均载荷

$$
\mathrm{MCF} \cdot 2 H=\frac{1}{\eta}\left(2 \pi M_{0} B+384 M_{0} \frac{H^{2}}{T}\right)
$$

根据静态平衡条件 ${ }^{[6]}$, 假设不同褶皱的波长 $2 H$ 是相同的

$$
\frac{\partial \mathrm{MCF}}{\partial H}=0
$$

故式(14)求偏导数可得

$$
H=0.072 \pi^{0.5} B^{0.5} T^{0.5}
$$

$B$ 为 $\mathrm{WMCT}$ 的拐角单元的胞壁边长之和, 将 $B$ 用内层壁边长 $D$ 和壁间距比 $N$ 进行表示

$$
B=480+2 D+2 \frac{D N+60}{N+1}
$$

从而得到由参数 $T, D, N$ 来表示的 $\mathrm{WMCT}$ 的 平均载荷在准静态条件下的数学模型为

$$
\mathrm{MCF}=6.928 \frac{\pi^{0.5} \sigma_{0} T^{1.5}}{\eta} \sqrt{480+2 D+2 \frac{D N+60}{N+1}}
$$

文中有效压缩距离系数 $\eta$ 的取值为 $0.7^{[2]}$ 。

为了获得 $\mathrm{MCF}$ 在动态载荷下的理论模型, 文 中进一步用动态系数 $\lambda$ 来修正式(18) ${ }^{[19]}$ 。表 4 给出 了具有不同几何参数的 $\mathrm{WMCT}$ 的动态与准静态 $\mathrm{MCF}$, 由表可知, 动态 $\mathrm{MCF}$ 与静态 $\mathrm{MCF}$ 之间的修 正系数 $\lambda$ 值约为 1.1 。

表 4 动态与准静态下的平均载荷对比表

\begin{tabular}{ccccccc}
\hline 组别 & $\begin{array}{c}\text { 内层壁边长 } \\
/ \mathrm{mm}\end{array}$ & $\begin{array}{c}\text { 壁厚 } \\
/ \mathrm{mm}\end{array}$ & $\begin{array}{c}\text { 壁间 } \\
\text { 距比 }\end{array}$ & $\begin{array}{c}\mathrm{MCF} \text { 准静态 } \\
/ \mathrm{kN}\end{array}$ & $\begin{array}{c}\mathrm{MCF} \text { 动态 } \\
/ \mathrm{kN}\end{array}$ & $\begin{array}{c}\text { 动态 } \\
\text { 系数 }\end{array}$ \\
\hline 1 & 20 & 0.9 & 1 & 60.972 & 63.281 & 1.04 \\
2 & 20 & 1.5 & 1 & 127.593 & 136.494 & 1.07 \\
3 & 10 & 0.9 & 1 & 56.699 & 62.805 & 1.11 \\
4 & 20 & 0.9 & 2 & 53.623 & 59.435 & 1.11 \\
\hline
\end{tabular}

MCF 的动态理论预测公式为

$$
\mathrm{MCF}=6.928 \frac{\lambda \pi^{0.5} \sigma_{0} T^{1.5}}{\eta} \sqrt{480+2 D+2 \frac{D N+60}{N+1}}
$$

比吸能 SEA 的理论预测公式也可表示为 


$$
\text { SEA }=4.409 \frac{\lambda \pi^{0.5} \sigma_{0} T^{0.5}}{\rho \eta \sqrt{480+2 D+2 \frac{D N+60}{N+1}}}
$$

\section{4 理论预测结果与讨论}

根据式(19)、(20), 表 5 7 分别给出在不同 $T(D=10 \mathrm{~mm}, N=1) 、 D(T=1.2 \mathrm{~mm}, N=1) 、 N(T=1.2$ $\mathrm{mm}, D=20 \mathrm{~mm}$ )下理论预测模型的预测值与有限元 分析值。由表可知, 在各个不同的参数中, 理论模 型都能较好地预测 WMCT 的 MCF 和 SEA, 最大误 差仅为 $11.9 \%$, 从而表明所推导的理论模型是具有 好的预测精度。

\section{表 $5 \mathrm{MCF}$ 和 SEA 理论和仿真值随 $\boldsymbol{T}$ 值的变化表}

\begin{tabular}{cccccc}
\hline $\begin{array}{c}\text { 壁厚 } \\
/ \mathrm{mm}\end{array}$ & $\begin{array}{c}\mathrm{MCF} \\
\text { 理论 } / \mathrm{kN}\end{array}$ & $\begin{array}{c}\mathrm{MCF} \\
\text { 仿真 } / \mathrm{kN}\end{array}$ & $\begin{array}{c}\mathrm{SEA} \text { 理论 } \\
/(\mathrm{kJ} / \mathrm{kg})\end{array}$ & $\begin{array}{c}\mathrm{SEA} \text { 仿真 } \\
/(\mathrm{kJ} / \mathrm{kg})\end{array}$ & $\begin{array}{c}\text { 误差 } \\
(\%)\end{array}$ \\
\hline 0.6 & 32.073 & 32.860 & 20.998 & 21.497 & 2.4 \\
0.9 & 58.923 & 63.281 & 25.718 & 27.616 & 7.4 \\
1.2 & 90.717 & 98.193 & 29.696 & 32.149 & 8.3 \\
1.5 & 126.781 & 136.494 & 33.201 & 35.745 & 7.7 \\
1.8 & 166.658 & 176.197 & 36.370 & 38.447 & 5.7 \\
\hline
\end{tabular}

表 $6 \mathrm{MCF}$ 和 SEA 理论和仿真值随 $D$ 值的变化表

\begin{tabular}{cccccc}
\hline $\begin{array}{c}\text { 内层壁 } \\
\text { 边长 } / \mathrm{mm}\end{array}$ & $\begin{array}{c}\mathrm{MCF} \\
\text { 理论 } / \mathrm{kN}\end{array}$ & $\begin{array}{c}\mathrm{MCF} \\
\text { 仿真 } / \mathrm{kN}\end{array}$ & $\begin{array}{c}\mathrm{SEA} \text { 理论 } \\
/(\mathrm{kJ} / \mathrm{kg})\end{array}$ & $\begin{array}{c}\mathrm{SEA} \text { 仿真 } \\
/(\mathrm{kJ} / \mathrm{kg})\end{array}$ & $\begin{array}{c}\text { 误差 } \\
(\%)\end{array}$ \\
\hline 5 & 87.249 & 91.137 & 30.876 & 32.253 & 4.5 \\
10 & 88.420 & 98.662 & 30.468 & 33.904 & 11.3 \\
15 & 89.576 & 100.204 & 30.074 & 33.642 & 11.9 \\
20 & 90.717 & 98.193 & 29.696 & 32.149 & 8.3 \\
25 & 91.844 & 96.738 & 29.332 & 30.892 & 5.3 \\
30 & 92.958 & 88.466 & 28.980 & 27.584 & -4.8 \\
\hline
\end{tabular}

表 7 MCF 和 SEA 理论和仿真值随 $N$ 值的变化

\begin{tabular}{cccccc}
\hline 壁间距比 & $\begin{array}{c}\mathrm{MCF} \\
\text { 理论 } / \mathrm{kN}\end{array}$ & $\begin{array}{c}\mathrm{MCF} \\
\text { 仿真 } / \mathrm{kN}\end{array}$ & $\begin{array}{c}\mathrm{SEA} \text { 理论 } \\
/(\mathrm{kJ} / \mathrm{kg})\end{array}$ & $\begin{array}{c}\mathrm{SEA} \text { 仿真 } \\
/(\mathrm{kJ} / \mathrm{kg})\end{array}$ & $\begin{array}{c}\text { 误差 } \\
(\%)\end{array}$ \\
\hline 0.5 & 91.720 & 90.667 & 29.372 & 29.033 & -1.2 \\
0.75 & 91.148 & 97.452 & 29.556 & 31.596 & 6.9 \\
1 & 90.717 & 98.193 & 29.696 & 32.149 & 8.3 \\
1.5 & 90.111 & 96.893 & 29.896 & 32.145 & 7.5 \\
2 & 89.704 & 94.298 & 30.032 & 31.568 & 5.1 \\
3 & 89.193 & 85.259 & 30.204 & 28.873 & -4.4 \\
\hline
\end{tabular}

\section{4 结论}

通过采用试验测试、数值分析与理论预测的方 法研究了窗型多壁结构的耐撞性。研究得到的几点 结论概况如下。

（1）窗型多壁结构的耐撞性优于同质量的方形 均匀多胞结构和方形三壁结构, 多壁设计能有效提 高碰撞效率及比吸能。

（2）壁厚 $T$ 、内层壁边长 $D$ 以及壁间距比 $N$ 对
窗型多壁结构的耐撞性能有重要的影响。

（3）窗型多壁结构的理论模型能较精确地预测 $\mathrm{MCF}$ 与 $\mathrm{SEA}$, 预测最大误差为 $11.9 \%$ 。

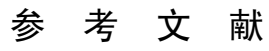

[1] 张立玲, 高峰. 金属薄壁吸能结构耐撞性研究进展 [J]. 机械工人：热加工，2006(1): 76-78.

ZHANG Liling, GAO Feng. Crashworthiness research progress of thin-walled energy-absorbing metal structures[J]. Mechanical Workers: Hot Working, 2006 (1): 76-78.

[2] WIERZBICKI T, ABRAMOWICZ W. On the crushing mechanics of thin-walled structures[J]. Journal of Applied Mechanics, 1983, 50(4): 727-734.

[3] ABRAMOWICZ W, JONES N. Dynamic progressive buckling of circular and square tubes[J]. International Journal of Impact Engineering， 1986，4(4): 243-270.

[4] GUILLOW S R, LU G, GRZEBIETA R H. Quasi-static axial compression of thin-walled circular aluminium tubes[J]. International Journal of Mechanical Sciences, 2001, 43(9): 2103-2123.

[5] CHEN W , WIERZBICKI T. Relative merits of single-cell, multi-cell and foam-filled thin-walled structures in energy absorption[J]. Thin-Walled Structures, 2001，39(4): 287-306.

[6] ZHANG Xiong, CHENG Gengdong, ZHANG Hui. Theoretical prediction and numerical simulation of multi-cell square thin-walled structures[J]. Thin-Walled Structures, 2006, 44: 1185-1191.

[7] JUSUF A, DIRGANTARA T, GUNAWAN L, et al. Crashworthiness analysis of multi-cell prismatic structures[J]. International Journal of Impact Engineering, 2015, 78(78): 34-50.

[8] WANG Zhonggang, LIU Jiefu, YAO Song. On folding mechanics of multi-cell thin-walled square tubes[J]. Composites Part B: Engineering, 2018，132(1): 17-27.

[9] WU Suzhen, ZHENG Gang, SUN Guangyong, et al. On design of multi-cell thin-wall structures for crash worthiness[J]. International Journal of Impact Engineering, 2016, 88: 102-117.

[10] WIERZBICKI T, JONES N. Structural failure[M]. Hoboken: Wiley, 1989.

[11] NIA A A, PARSAPOUR M. An investigation on the energy absorption characteristics of multi-cell square tubes[J]. Thin-Walled Structures, 2013，68(10): 26-34.

[12] 文桂林, 孔祥正. 泡沫填充夹芯墙多胞结构的耐撞性多 目标优化设计 [J]. 振动与冲击, 2015，34(24105): 115-121. 
WEN Guilin, KONG Xiangzheng, et al. Multi-objective crashworthiness optimization design of foam-filled sandwich wall multi-cell structures[J]. Journal of Vibration and Shock, 2015， 34 (24105): 115-121.

[13] 张勇, 刘舒然, 赖雄鸣, 等. 泡沫填充薄壁结构的抗弯 性分析及多目标优化设计 [J]. 机械工程学报, 2016, 52(24): 115-122.

ZHANG Yong, LIU Shuran, LAI Xiongming, et al. Bending resistance analysis and multi-objective optimization design of foam filled thin-walled structure[J]. Journal of Mechanical Engineering, 2016， 52(24): 115-122.

[14] 兰风崇, 马聪承, 陈吉清, 等. 泡沫铝填充分体式翻转 结构设计与优化分析 [J]. 机械工程学报, 2017, 53(12): 156-165.

LAN Fengchong, MA Congcheng, CHEN Jiqing, YU Jianli, et al. Structural design optimization of split typed flip tubes filled with aluminum foam[J]. Journal of Mechanical Engineering, 2017, 53(12): 156-165.

[15] NHAN T T. Theoretical prediction and optimization for crashworthiness of multi-cell tubes under impact loading[D]. Changsha: Hunan University, 2015.

[16] QIU Na, GAO Yunkai, FANG Jiangguang, et al.
Theoretical prediction and optimization of multi-cell hexagonal tubes under axial crashing[J]. Thin-Walled Structures, 2016, 102: 111-121.

[17] ZHANG Xiong, ZHANG Hui. Energy absorption of multi-cell stub columns under axial compression[J]. Thin-Walled Structures, 2013，68(10): 156-163.

[18] ZHANG Xiong, ZHANG Hui. Numerical and theoretical studies on energy absorption of three-panel angle elements[J]. International Journal of Impact Engineering, 2012, 46(6): 23-40.

[19] HE Qiang, MA Dawei, ZHANG Zhedong, et al. Mean compressive stress constitutive equation and crashworthiness optimization design of three novel honeycombs under axial compression[J]. International Journal of Mechanical Sciences, 2015， 99: 274-287.

作者简介: 何宁, 男, 1994 年出生。主要研究方向为先进机械与车辆优 化设计。

E-mail: 364644660@qq.com

张勇(通信作者), 男, 1980 年出生, 教授。主要研究方向为轻质材料的 耐撞性研究。

E-mail: zhangyong@hqu.edu.cn

陈腾腾, 男, 1994 年出生。主要研究方向为先进机械与车辆优化设计。 E-mail: 1013123777@qq.com 